\title{
THE STRUCTURE OF MULTIVARIATE POISSON DISTRIBUTION
}

\author{
By KazUTOMO KaWAMURA
}

\section{Summary}

In this paper we shall derive a multivariate Poisson distribution and we shall discuss its structure.

\section{Notations and Definitions}

$\begin{array}{ll}n & \text { positive integer } \\ \boldsymbol{X}=\left(X_{1}, X_{2}, \cdots, X_{n}\right) & n \text { dimensional random vector } \\ \boldsymbol{i}=\left(l_{1}, i_{2}, \cdots, i_{n}\right) & n \text { dimensional vector with } 0,1 \text { components } \\ \boldsymbol{k}=\left(k_{1}, k_{2}, \cdots, k_{n}\right) & n \text { dimensional vector with nonnegative integer } \\ & \begin{array}{l}\text { components } \\ \boldsymbol{x}=\left(x_{1}, x_{2}, \cdots, x_{n}\right)\end{array} \\ p(x, \lambda) & \text { observation of } \boldsymbol{X} \\ & \text { Poisson density with parameter } \lambda\end{array}$

\section{Main Results}

1. Multivariate Bernoulli distribution $B\left(1, p_{i}\right)$

Multivariate Bernoulli distribution is defined by

where $p_{i} \geqq 0$ and $\Sigma_{\imath} p_{i}=1$.

$$
P(\boldsymbol{X}=\boldsymbol{i})=p_{\boldsymbol{i}}
$$

The moment generating function (m.g. f.) is given by

$$
g(\boldsymbol{s})=\sum_{\imath} p_{i} s_{1}{ }^{{ }^{2}} S_{2}{ }^{{ }_{2} \cdots} \cdots s_{n}{ }^{{ }^{2} n} .
$$

The marginal distribution of this multivariate Bernoulli distribution is also degenerated Bernoulli.

The covariance matrix of $B\left(1, p_{i}\right)$ is given by

$$
\begin{aligned}
& \operatorname{Cov}\left(X_{j}, X_{k}\right)=\sum_{\imath_{j}=\imath_{k}=0} p_{i} \sum_{\imath_{j}=\imath_{k}=1} p_{i}-\sum_{\imath_{j}=1, \imath_{k}=0} p_{i} \sum_{\imath_{j}=0, \imath_{k}=1} p_{i}, \\
& \operatorname{Var}\left(X_{j}\right)=\sum_{\imath_{j}=0} p_{i} \sum_{\imath_{j}=1} p_{i} .
\end{aligned}
$$

Received May 18, 1978 
Proof.

$$
\begin{aligned}
\operatorname{Cov}\left(X_{j}, X_{k}\right)= & \sum_{\imath=\imath_{k}=1} x_{j} x_{k} p_{i}-\sum_{\imath_{j}=1} x_{j} p_{i} \sum_{\imath_{k}=1} x_{k} p_{i} \\
= & \sum_{\imath_{j}=\imath_{k}=1} p_{i}-\sum_{\imath_{j}=1} p_{i} \sum_{\imath_{k}=1} p_{i} \\
= & \sum_{\imath_{j}=\imath_{k}=1} p_{i}\left(\sum_{\imath_{j}=\imath_{k}=0} p_{i}+\sum_{\imath_{j}=1, \imath_{k}=0} p_{i}+\sum_{\imath_{j}=0, \imath_{k}=1} p_{i}+\sum_{\imath_{j}=\imath_{k}=1} p_{i}\right) \\
& -\left(\sum_{\imath_{j}=1, \imath_{k}=0} p_{i}+\sum_{\imath \jmath} \sum_{\imath_{k}=1} p_{i}\right)\left(\sum_{\imath_{j}=0, \imath_{k}=1} p_{i}+\sum_{\imath_{j}=\imath_{k}=1} p_{i}\right) \\
= & \sum_{\imath_{j}=\imath_{k}=0} p_{i} \sum_{\imath_{j}=\imath_{k}=1} p_{i}-\sum_{\imath_{j}=1, \imath_{k}=0} p_{i} \sum_{\imath_{j}=0, \imath_{k}=1} p_{i} . \\
\operatorname{Var}\left(X_{j}\right)= & \sum_{\imath_{j}=1} x_{j}{ }^{2} p_{i}-\left(\sum_{\imath_{j}=1} x_{j} p_{i}\right)^{2} \\
= & \sum_{\imath_{j}=1} p_{i}-\left(\sum_{\imath_{j}=1} p_{i}\right)^{2} .
\end{aligned}
$$

2. Multivariate binomial distribution $B\left(N, p_{i}\right)$

Multivariate binomial distribution is defined by

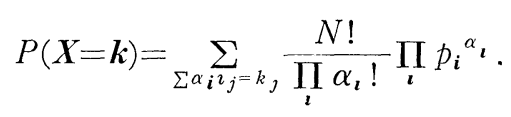

This distribution is derived by $N$ time convolution of $B\left(1, p_{i}\right)$. The m.g.f. of the distribution is given by

$$
g_{N}(\boldsymbol{s})=\left(\sum_{\imath} p_{i} s_{1}{ }^{2} S_{2}{ }^{2} \cdots \cdots s_{n}{ }^{2}\right)^{N} .
$$

The marginal distribution of this multivariate binomial distribution is also degenerated binomial.

Covariance matrix of $B\left(N, p_{i}\right)$ is given by

$$
\begin{aligned}
& \operatorname{Cov}\left(X_{\jmath}, X_{k}\right)=N\left(\sum_{\imath,=\imath_{k}=0} p_{i} \sum_{\imath \jmath=\imath_{k}=1} p_{i}-\sum_{\imath_{j}=1, \imath_{k}=0} p_{i} \sum_{\imath_{j}=0, \imath_{k}=1} p_{\imath}\right), \\
& \operatorname{Var}\left(X_{j}\right)=N\left(\sum_{\imath=0} p_{i} \sum_{\imath_{j}=1} p_{i}\right) .
\end{aligned}
$$

3. Multivariate Poisson distribution

Multivariate Poisson distribution is a limiting distribution of $B\left(N, p_{\boldsymbol{i}}\right)$ as $N \rightarrow \infty$ under the condition of $N p_{i}=\lambda_{\imath}$ where $\lambda_{\imath}$ is a non-negative fixed parameter. If a random vector $\boldsymbol{X}$ has a binomial distribution $B\left(N, p_{i}\right)$ and if we assume $N p_{i}=\lambda_{\boldsymbol{l}}$ then we have

$$
\lim _{N \rightarrow \infty} P(\boldsymbol{X}=\boldsymbol{k})=\sum_{\sum \alpha_{\boldsymbol{i}} \imath_{j}=k,} \prod_{\boldsymbol{i} \neq 0} p\left(\alpha_{\boldsymbol{\imath}}, \lambda_{\boldsymbol{\imath}}\right)
$$

where $p\left(\alpha_{\imath}, \lambda_{\imath}\right)$ is an univariate Poisson density.

THEOREM 1. If a random vector $\boldsymbol{X}$ has a distribution $B\left(N, \phi_{i}\right)$ then we have 
THE STRUCTURE OF MULTIVARIATE POISSON DISTRIBUTION

$$
\lim _{N p_{i}=\lambda_{\imath}, N \rightarrow \infty} P(\boldsymbol{X}=\boldsymbol{k})=\sum_{\sum \alpha_{\boldsymbol{i}} \imath_{\jmath}=k,} \prod_{\boldsymbol{i} \neq 0} p\left(\alpha_{\imath}, \lambda_{\imath}\right),
$$

Proof. By the condition we get

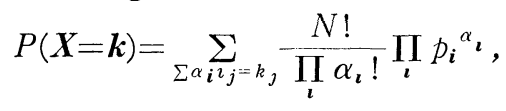

therefore the limit value of each term is

$$
\begin{aligned}
& \lim _{N p_{i}=\lambda_{\imath}, N \rightarrow \infty} \frac{N !}{\prod_{i} \alpha_{\imath} !} \prod_{\imath} p_{i}{ }^{\alpha} \\
& =\lim \frac{N !}{\alpha_{0} ! \prod_{\imath \neq 0} \alpha_{\imath} !}\left(1-\frac{\sum_{i \neq 0} \lambda_{\imath}}{N}\right)^{\alpha_{0}} \prod_{\imath \neq 0} \frac{\lambda_{\imath}{ }^{\alpha_{\imath}}}{N^{\alpha}{ }_{\imath}} \\
& =\lim \frac{N !}{\alpha_{0} ! N \sum_{i \neq 0}^{\sum_{0} \alpha_{i}}} \lim \left(1-\frac{\sum_{i \neq 0} \lambda_{\imath}}{N}\right)^{N-\sum_{i \neq 0} \alpha_{i}} \prod_{\imath \neq 0} \lambda_{\imath}{ }^{\alpha} \iota / \prod_{\imath \neq 0} \alpha_{\imath} \text { ! } \\
& =\exp \left\{-\sum_{\boldsymbol{\imath} \neq 0} \lambda_{i}\right\} \prod_{\boldsymbol{\imath} \neq 0} \lambda_{\imath}{ }^{a} \imath / \prod_{\boldsymbol{\imath} \neq 0} \alpha_{\imath} ! \\
& =\prod_{\imath \neq 0} p\left(\alpha_{\imath}, \lambda_{\imath}\right) \text {. }
\end{aligned}
$$

Therefore we have

$$
\begin{aligned}
& \lim _{N p_{\boldsymbol{i}}=\lambda_{\boldsymbol{\imath}}, N \rightarrow \infty} P(\boldsymbol{X}=\boldsymbol{k})=\lim _{N p_{\boldsymbol{i}}=\lambda_{\boldsymbol{\imath}}, N \rightarrow \infty} \sum_{\sum \alpha_{\boldsymbol{i}} \imath_{j}=k_{j}} N ! \prod_{\boldsymbol{\imath}} \frac{p_{\boldsymbol{i}}{ }^{\alpha} \boldsymbol{\imath}}{\alpha_{\boldsymbol{\imath}} !} \\
& =\sum_{\sum \alpha_{i} \imath_{j}=k_{j}} \exp \left\{-\sum_{\boldsymbol{i} \neq 0} \lambda_{i}\right\} \prod_{\boldsymbol{\imath} \neq 0} \frac{\lambda_{\boldsymbol{\imath}}{ }_{\boldsymbol{\imath}}}{\alpha_{\boldsymbol{\imath}} !} \\
& =\sum_{\sum \alpha_{i} \imath j=k} \prod_{i \neq 0} p\left(\alpha_{\imath}, \lambda_{\imath}\right) \text {. }
\end{aligned}
$$

THEOREM 2. The moment generating function of the limiting distribution is given by

$$
\begin{aligned}
h(\boldsymbol{s}) & =\exp \left\{-\sum_{\boldsymbol{i} \neq 0} \lambda_{i}+\sum_{\boldsymbol{i} \neq 0} \lambda_{i} \boldsymbol{s}^{i}\right\} \\
& =\prod_{\boldsymbol{\imath} \neq 0} \exp \left\{-\lambda_{i}+\lambda_{i} \boldsymbol{s}^{i}\right\} .
\end{aligned}
$$

Proof.

$$
\begin{aligned}
& h(\boldsymbol{s})=\lim _{N p_{\boldsymbol{i}}=\lambda_{\boldsymbol{\imath}}, N \rightarrow \infty} g(\boldsymbol{s})^{N}=\lim _{N p_{\boldsymbol{i}}=\lambda_{\boldsymbol{\imath}}, N \rightarrow \infty}\left(\sum_{\boldsymbol{i}} p_{\boldsymbol{i}} S_{1}{ }^{{ }_{1}} S_{2}{ }^{2} \ldots S_{n}{ }^{2}\right)^{N} \\
& =\lim _{N \rightarrow \infty}\left(1-\sum_{i \neq 0} p_{i}+\sum_{i \neq 0} p_{i} s^{i}\right)^{N} \\
& =\lim _{N \rightarrow \infty}\left(1-\sum_{\boldsymbol{\imath} \neq 0} p_{i}+\sum_{\boldsymbol{\imath} \neq 0} \frac{\lambda_{\boldsymbol{l}}}{N} \boldsymbol{s}^{\boldsymbol{\imath}}\right)^{N} \\
& =\lim _{N \rightarrow \infty}\left(1-\sum_{\boldsymbol{i} \neq 0} \frac{\lambda_{i}}{N}+\sum_{i \neq 0} \frac{\lambda_{i}}{N} \boldsymbol{s}^{\boldsymbol{\imath}}\right)^{N}
\end{aligned}
$$




$$
=\exp \left\{-\sum_{i \neq 0} \lambda_{i}+\sum_{i \neq 0} \lambda_{i} \boldsymbol{s}^{i}\right\}
$$

where $\boldsymbol{s}^{\boldsymbol{\imath}}={S_{1}}^{\boldsymbol{t}_{1}} S_{2}{ }^{\boldsymbol{t}_{2}} \cdots{S_{n}}^{{ }^{2} n}$.

THEOREM 3. If a random vector $\boldsymbol{X}$ has the Porsson law, then we have an unique decomposition of the random vector by $X_{j}=\sum_{\imath_{j}=1} X_{\boldsymbol{\imath}}$ where $X_{\boldsymbol{i}}(\boldsymbol{i} \neq \mathbf{0})$ are mutually independent, unvvariate Poisson variables with parameter $\lambda_{\imath}$.

Proof. This is a direct conclusion from the definition of the distribution of convolution type. Mathematical proof is given as followings. In the bivariate case $n=2$, if $\boldsymbol{X}=\left(X_{1}, X_{2}\right)$ has the Poisson law, our m.g.f. $h(\boldsymbol{s})$ becomes

$$
\begin{aligned}
h(\boldsymbol{s}) & =\exp \left\{-\left(\lambda_{10}+\lambda_{01}+\lambda_{11}\right)+\lambda_{10} s_{1}+\lambda_{01} s_{2}+\lambda_{11} s_{1} s_{2}\right\} \\
& =\exp \left(-\lambda_{10}+\lambda_{10} s_{1}\right) \exp \left(-\lambda_{01}+\lambda_{01} s_{2}\right) \exp \left(-\lambda_{11}+\lambda_{11} s_{1} s_{2}\right) .
\end{aligned}
$$

If we put $s_{2}=1$, then we get the m. g.f. of $X_{1}$

$$
\exp \left(-\lambda_{10}+\lambda_{10} s_{1}\right) \exp \left(-\lambda_{11}+\lambda_{11} s_{1}\right) .
$$

This is a m.g.f. of convolution type, and if we put $s_{1}=1$, we get

$$
\exp \left(-\lambda_{01}+\lambda_{01} s_{2}\right) \exp \left(-\lambda_{11}+\lambda_{11} s_{2}\right)
$$

the m.g.f. of $X_{2}$ of convolution type. Then we have a decomposition

$$
X_{1}=X_{10}+X_{11}{ }^{\prime}, \quad X_{2}=X_{01}+X_{11}^{\prime \prime} \text {. }
$$

If we put $X_{11}{ }^{\prime} \neq X_{11}{ }^{\prime \prime}$ with positive probability then this is contradictory to the fact that $X_{11}^{\prime}, X_{11}^{\prime \prime}$ has a bivariate m.g.f.

$$
\exp \left(-\lambda_{11}+\lambda_{11} s_{1} s_{2}\right)
$$

Therefore we have $X_{11}{ }^{\prime}=X_{11}{ }^{\prime \prime}=X_{11}$ with probability one. And we can express the decomposition of given $X=\left(X_{1}, X_{2}\right)$ as

$$
X_{1}=X_{10}+X_{11}, \quad X_{2}=X_{01}+X_{11} .
$$

In this equality $X_{10}, X_{01}$ and $X_{11}$ are mutually independent Poisson distribution with parameter $\lambda_{10}, \lambda_{01}$ and $\lambda_{11}$ respectively, as to be proved.

Theorem 4. The covariance matrix of the multivarnate Poisson distribution is given by

$$
\operatorname{Var}\left(X_{j}\right)=\sum_{\imath_{j}=1} \lambda_{\imath}, \quad \operatorname{Cov}\left(X_{\jmath}, X_{k}\right)=\sum_{\imath_{j}=\imath_{k}=1} \lambda_{\imath}
$$

Proof. This is a direct conclusion using the m.g.f. of the distribution (Theorem 2) or the preceding decomposition Theorem. Generally a m.g.f. is given by 


$$
h(\boldsymbol{s})=\sum_{\boldsymbol{k}} \boldsymbol{s}^{k} P(\boldsymbol{X}=\boldsymbol{k}) .
$$

And we have

$$
\begin{aligned}
& \frac{\partial^{2} h(\boldsymbol{s})}{\partial s_{i} \partial s_{\jmath}}=\sum_{\boldsymbol{k}} k_{i} k_{j} s_{1}{ }^{{ }_{1} \cdots} \cdots s_{\imath}{ }^{k_{i}-1} \cdots s_{\jmath}{ }^{{ }{ }^{-1} \cdots} \cdots s_{n}{ }^{k} n P(\boldsymbol{X}=\boldsymbol{k}), \\
& {\left[\frac{\partial^{2} h(s)}{\partial s_{i} \partial s_{\jmath}}\right]_{s_{1}=s_{2}=\cdots=s_{n}=1}=\sum_{k} k_{i} k_{j} P(\boldsymbol{X}=\boldsymbol{k})=E\left(X_{\imath} X_{\jmath}\right) .}
\end{aligned}
$$

We use the result of Theorem $2, h(s)$ becomes

$$
h(s)=\exp \left\{-\sum_{i \neq 0} \lambda_{i}+\sum_{i \neq 0} \lambda_{i} s^{i}\right\},
$$

then

$$
\begin{aligned}
& \frac{\partial h(s)}{\partial s_{k}}=h(\boldsymbol{s})\left\{\sum_{\boldsymbol{i} \neq 0} \imath_{k} \lambda_{i} s_{1}{ }^{{ }_{1} 1 \cdots} s_{k}{ }^{{ }^{2}{ }^{2}-1} \cdots s_{n}{ }^{{ } n}\right\} \\
& \frac{\partial^{2} h(\boldsymbol{s})}{\partial s_{j} \partial s_{k}}=\frac{\partial h(\boldsymbol{s})}{\partial s_{\jmath}}\left\{\sum_{i \neq 0} \imath_{k} \lambda_{i} s_{1}{ }^{{ }_{1}} \cdots s_{k}{ }^{{ }^{2}-1} \cdots s_{n}{ }^{{ } n}\right\} \\
& +h(\boldsymbol{s}) \frac{\partial}{\partial s_{\jmath}}\left\{\sum_{\boldsymbol{i} \neq 0} \imath_{k} \lambda_{i} s_{1}{ }^{2} \cdots s_{k}{ }^{2}{ }^{2}-1 \cdots s_{n}{ }^{2} n\right\}
\end{aligned}
$$

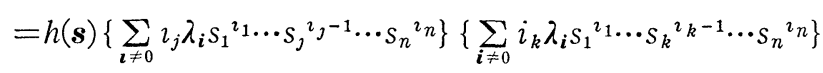

$$
\begin{aligned}
& +h(\boldsymbol{s})\left\{\sum_{\boldsymbol{i} \neq 0} \imath_{j} \imath_{k} \lambda_{i} s_{1}{ }^{2} \cdots \cdots S_{\jmath}{ }^{2} \jmath-1 \cdots s_{k}{ }^{2}{ }^{2}-1 \cdots \cdot s_{n}{ }^{2} n\right\} \\
& {\left[\frac{\partial^{2} h(s)}{\partial s_{j} \partial s_{k}}\right]_{s_{1}=s_{2}=\cdots=s_{n}=1}} \\
& =\left.h(\boldsymbol{s})\right|_{s_{1}=s_{2}=\cdots=s_{n}=1}\left[\left(\sum_{i \neq 0} \imath_{j} \lambda_{\boldsymbol{l}}\right)\left(\sum_{\boldsymbol{i} \neq 0} \imath_{k} \lambda_{\boldsymbol{l}}\right)+\sum_{\boldsymbol{i} \neq 0} i_{j} i_{k} \lambda_{\boldsymbol{l}}\right] \\
& E\left(X, X_{k}\right)=\sum_{\imath_{j}=\imath v_{k}=1} \lambda_{i}+\left(\sum_{\imath_{j}=1} \lambda_{\imath}\right)\left(\sum_{\imath_{k}=1} \lambda_{\imath}\right) .
\end{aligned}
$$

And $E\left(X_{j}\right), E\left(X_{k}\right)$ is given by $E\left(X_{j}\right)=\operatorname{Var}\left(X_{j}\right)=\sum_{\imath=1} \lambda_{\imath}, E\left(X_{k}\right)=\operatorname{Var}\left(X_{k}\right)=\sum_{\imath=1} \lambda_{\imath}$.

Finally we have

$$
\begin{aligned}
& \operatorname{Cov}\left(X_{\jmath}, X_{k}\right)=E\left(X_{\jmath} X_{k}\right)-E\left(X_{j}\right) E\left(X_{k}\right)=\sum_{\imath_{j}=\imath_{k}=1} \lambda_{l}, \\
& \operatorname{Var}\left(X_{j}\right)=E\left(X_{j}\right)=\sum_{\imath_{j}=1} \lambda_{l} .
\end{aligned}
$$

THEOREM 5. If a random vector $\boldsymbol{X}$ has the Porsson law, then the marginal distribution is also a degenerated Porsson.

Proof. Since $\boldsymbol{X}$ has a m. g. f. $h(\boldsymbol{s})$, it follows that $\boldsymbol{X}^{(\jmath)}=\left(X_{1}, \cdots, X_{\jmath-1}, X_{\jmath+1}\right.$, $\left.\cdots, X_{n}\right)(\jmath=1,2, \cdots, n)$ has a m.g.f. $\left.h(s)\right|_{s_{j}=1}$. 


$$
\begin{aligned}
& \left.h(\boldsymbol{s})\right|_{s_{j}=1}=\exp \left\{-\sum_{\boldsymbol{\imath} \neq 0} \lambda_{i}+\sum_{\boldsymbol{\imath} \neq 0} \lambda_{i} \boldsymbol{s}^{i}\right\}_{s_{j=1}} \\
& =\exp \left\{-\sum_{i(j) \neq 0}\left(\sum_{i j=0,1} \lambda_{l}\right)+\sum_{i(j) \neq 0}\left(\sum_{i j=0,1} \lambda_{\imath}\right) s_{1}{ }^{2}{ }^{2} \cdots s_{j-1}{ }^{\imath \jmath} j-1 S_{j+1}{ }^{2} \jmath+1 \cdots s_{n}{ }^{2} n\right\} .
\end{aligned}
$$

This means that if $\boldsymbol{X}$ has a Poisson distribution, it follows that $\boldsymbol{X}^{(j)}$ has also a generated Poisson distribution. And, similarly, if we put

$$
\boldsymbol{X}^{\left(\jmath_{1}, \jmath_{2}, \cdots, \jmath_{k}\right)}=\left(X_{1}, \cdots, X_{\jmath_{1}-1}, X_{\jmath_{1}+1}, \cdots, X_{\jmath_{2}-1}, X_{\jmath_{2}+1}, \cdots, X_{\jmath_{k}-1}, X_{\jmath_{k}+1}, \cdots, X_{n}\right)
$$

then the m.g.f. of the vector is given by

$$
\begin{aligned}
& \left.h(\boldsymbol{s})\right|_{s_{\jmath_{1}=s_{j_{2}}=\cdots=s_{j_{k}=1}}}
\end{aligned}
$$

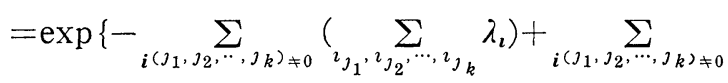

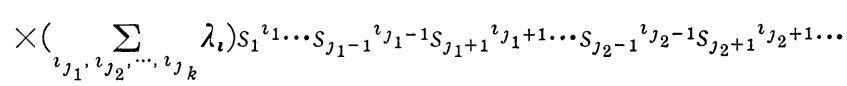

$$
\begin{aligned}
& \left.s_{J_{k}-1}{ }^{2}{ }_{k}-1 s_{J_{k}+1}{ }^{2} \jmath_{k}+1 \cdots S_{n}{ }^{2}\right\} \text {. }
\end{aligned}
$$

Therefore, the random vector $\boldsymbol{X}^{\left(\jmath_{1}, J_{2}, \cdots, \jmath_{k}\right)}$ has a degenerated Poisson distribution as to be proved.

Corollary 1. The marginal distribution $X_{j}$ of $\boldsymbol{X}$ is Poisson with a param$\operatorname{eter} \sum_{i_{j}=1} \lambda_{l}$.

Corollary 2. If $\operatorname{Cov}\left(X_{\jmath}, X_{k}\right)=0(\jmath \neq k)$, then $X$, and $X_{k}$ are mutually independent random variables.

THEOREM 6. If $\boldsymbol{X}_{1}, \boldsymbol{X}_{2}, \cdots, \boldsymbol{X}_{N}$ are mutually independent random vectors of the multvariate Poisson distribution, then the sum $\sum_{j=1}^{N} \boldsymbol{X}_{\jmath}$ has a multvariate Porsson distribution.

Proof. The m.g.f. of the sum vector is given by

$$
\begin{aligned}
h(\boldsymbol{s})^{N} & =\exp N\left\{-\sum_{\boldsymbol{i} \neq 0} \lambda_{i}+\sum_{\boldsymbol{i} \neq 0} \lambda_{i} \boldsymbol{s}^{i}\right\} \\
& =\exp \left\{-\sum_{\boldsymbol{i} \neq 0} N \lambda_{i}+\sum_{\boldsymbol{i} \neq 0} N \lambda_{i} \boldsymbol{s}^{i}\right\} .
\end{aligned}
$$

This means that the sum vector is also a multivariate Poisson distribution with parameter $N \lambda_{\imath}(\boldsymbol{i} \neq \mathbf{0})$.

\section{Estimation of covariance matrix}

We assume that $\boldsymbol{X}_{1}, \boldsymbol{X}_{2}, \cdots, \boldsymbol{X}_{N}$ are mutually independent multivariate Poisson random vectors with unknown parameter $\lambda_{2}$. Given a sequence of the 
random vectors, we shall estimate the mean vector and the covariance matrix, in this section.

\section{A sequence of multivariate Poisson random vectors}

$$
\boldsymbol{X}_{k}=\left(X_{1 k}, \cdots, X_{n k}\right) \quad(k=1,2, \cdots, N)
$$

$\begin{array}{rrrrrrrrrrrrrrrrrrrrrr}k & 1 & 2 & 3 & 4 & 5 & 6 & 7 & 8 & 9 & 10 & 11 & 12 & 13 & 14 & 15 & 16 & 17 & 18 & 19 & 20 & \text { sum } \\ X_{1 k} & 1 & 0 & 0 & 0 & 1 & 0 & 2 & 1 & 1 & 0 & 0 & 0 & 0 & 1 & 0 & 0 & 0 & 0 & 1 & 0 & 8 \\ X_{2 k} & 0 & 0 & 0 & 0 & 0 & 0 & 0 & 1 & 0 & 1 & 0 & 1 & 0 & 1 & 0 & 0 & 1 & 0 & 0 & 1 & 6 \\ X_{3 k} & 1 & 3 & 0 & 0 & 1 & 0 & 1 & 1 & 1 & 1 & 0 & 2 & 0 & 0 & 0 & 0 & 1 & 2 & 1 & 1 & 16 \\ X_{4 k} & 3 & 2 & 2 & 0 & 1 & 0 & 0 & 2 & 0 & 1 & 0 & 1 & 0 & 0 & 0 & 0 & 1 & 2 & 3 & 1 & 19 \\ X_{5 k} & 5 & 4 & 5 & 15 & 10 & 7 & 5 & 11 & 6 & 4 & 9 & 11 & 11 & 3 & 10 & 7 & 14 & 8 & 5 & 7 & 157 \\ X_{6 k} & 8 & 8 & 8 & 14 & 12 & 8 & 6 & 9 & 2 & 4 & 3 & 11 & 8 & 5 & 7 & 11 & 10 & 6 & 4 & 2 & 146 \\ X_{7 k} & 1 & 3 & 2 & 0 & 0 & 0 & 0 & 1 & 0 & 1 & 1 & 2 & 2 & 1 & 0 & 0 & 1 & 1 & 1 & 0 & 17 \\ X_{8 k} & 2 & 3 & 1 & 1 & 0 & 1 & 0 & 1 & 0 & 2 & 4 & 1 & 2 & 2 & 0 & 0 & 2 & 1 & 0 & 1 & 24 \\ \text { sum } & 21 & 23 & 18 & 30 & 25 & 16 & 14 & 27 & 10 & 14 & 17 & 29 & 23 & 13 & 17 & 18 & 30 & 20 & 15 & 13 & \end{array}$

This sequence of random vectors is of $n=8$ dimensional Poisson distribution and the sample size $N=20$. In this paper, we use

$$
\begin{aligned}
\bar{X}_{i}=\frac{1}{N} \sum_{k=1}^{N} X_{\imath k}, \quad S_{\imath \jmath} & =\frac{1}{N} \sum_{k=1}^{N}\left(X_{i k}-\bar{X}_{\imath}\right)\left(X_{j k}-\bar{X}_{j}\right) \\
& =\frac{1}{N} \sum_{k=1}^{N} X_{i k} X_{\jmath k}-\bar{X}_{\imath} \bar{X}_{\jmath} \quad(1 \leqq \imath, \jmath \leqq n),
\end{aligned}
$$

where we get easily $S_{\imath j}=S_{\jmath i}$.

\section{Estimated mean values and standard deviations}

$$
\begin{array}{llll} 
& \bar{X}_{\imath}, & S_{\imath}{ }^{2}=\frac{1}{N} \sum_{k=1}^{N}\left(X_{\imath k}-\bar{X}_{\imath}\right)^{2} & (\imath=1,2, \cdots, n) \\
\bar{X}_{1}=0.4 & \bar{X}_{2}=0.3 & \bar{X}_{3}=0.8 & \bar{X}_{4}=0.95 \\
S_{1}=0.5831 & S_{2}=0.4583 & S_{3}=0.8124 & S_{4}=1.0235 \\
\bar{X}_{5}=7.85 & \bar{X}_{6}=7.3 & \bar{X}_{7}=0.85 & \bar{X}_{8}=1.2 \\
S_{5}=3.3208 & S_{6}=3.2573 & S_{7}=0.8529 & S_{8}=1.0770
\end{array}
$$

Sample mean of the sum $\quad 19.65$

Standard deviation of the sum $\quad 5.9521$

\section{Estimated covariance matrix}

The estimated covariance matrix is given by 


$$
\begin{aligned}
S & =\left[S_{i j}\right] \\
& =\left(\begin{array}{rccccccr}
0.34 & -0.02 & 0.03 & 0.07 & -0.64 & -0.32 & -0.14 & -0.23 \\
-0.02 & 0.21 & 0.06 & 0.015 & 0.145 & -0.14 & 0.045 & 0.09 \\
0.03 & 0.06 & 0.66 & 0.44 & -0.43 & -0.09 & 0.32 & 0.09 \\
0.07 & 0.015 & 0.44 & 1.05 & -0.86 & -0.085 & 0.49 & 0.06 \\
-0.64 & 0.145 & -0.43 & -0.86 & 11.03 & 6.445 & -0.42 & -0.12 \\
-0.32 & -0.14 & -0.09 & -0.085 & 6.445 & 10.61 & 0.145 & -0.56 \\
-0.14 & 0.045 & 0.32 & 0.49 & -0.42 & 0.145 & 0.73 & 0.53 \\
-0.23 & 0.09 & 0.09 & 0.06 & -0.12 & -0.56 & 0.53 & 1.16
\end{array}\right)
\end{aligned}
$$

The main components

$$
S_{i i}=S_{i}{ }^{2}=\frac{1}{N} \sum_{k=1}^{N}\left(X_{i k}-\bar{X}_{\imath}\right)^{2} \quad(\imath=1,2, \cdots, n)
$$

will be refined by using estimated mean vector

$$
\bar{X}_{\imath}=\frac{1}{N} \sum_{k=1}^{N} X_{\imath k} \quad(\imath=1,2, \cdots, n) .
$$

And the estimated sample covariances in $S$ with negative values are not natural, because all parameters $\sum_{i_{i}=\imath_{j}=1} \lambda_{i}$ estimated by $S_{i j}$ must be nonnegative. Therefore we shall refine the estimeter $\boldsymbol{S}$ as $\boldsymbol{S}^{+}=\left[S_{\imath \jmath}{ }^{+}\right]$where $S_{\imath \jmath}{ }^{+}$equals to $S_{\imath \jmath}$ iff $S_{\imath j} \geqq 0$ and 0 iff $S_{\imath j}<0$. And a more refined estimater will be given by $\widetilde{\boldsymbol{S}}=\left[\widetilde{S}_{\imath \jmath}\right]$ where $\widetilde{S}_{\imath}$ is defined by

$$
\tilde{S}_{\imath j}=\left\{\begin{array}{lll}
\bar{X}_{\imath} & \text { iff } & i=\jmath, \\
S_{i j}{ }^{+} & \text {iff } & i \neq \jmath .
\end{array}\right.
$$

Because the parameter estimated by $S_{\imath \imath}$ is the variance value of $X_{\imath}$ and equals to the mean value of $X_{\imath}$. And we get easily $S_{\imath \imath}{ }^{+}=S_{\imath i} \geqq 0, S_{\imath j}{ }^{+}=S_{j i}{ }^{+}$and $\widetilde{S}_{\imath j}=\widetilde{S}_{j i}$. Then we have

$$
\widetilde{\boldsymbol{S}}=\left(\begin{array}{llllllll}
0.4 & 0 & 0.03 & 0.07 & 0 & 0 & 0 & 0 \\
0 & 0.3 & 0.06 & 0.015 & 0.145 & 0 & 0.045 & 0.09 \\
0.03 & 0.06 & 0.8 & 0.44 & 0 & 0 & 0.32 & 0.09 \\
0.07 & 0.015 & 0.44 & 0.95 & 0 & 0 & 0.49 & 0.06 \\
0 & 0.145 & 0 & 0 & 7.85 & 6.445 & 0 & 0 \\
0 & 0 & 0 & 0 & 6.445 & 7.3 & 0.145 & 0 \\
0 & 0.045 & 0.32 & 0.49 & 0 & 0.145 & 0.85 & 0.53 \\
0 & 0.09 & 0.09 & 0.06 & 0 & 0 & 0.53 & 1.2
\end{array}\right)
$$

Conclusion of this section.

1. The unknown mean values of $\left(X_{1}, \cdots, X_{n}\right)$ 
are to be estimated by

$$
E X_{1}=\sum_{\imath_{1}=1} \lambda_{\imath}, \cdots, E X_{n}=\sum_{\imath_{n}=1} \lambda_{\imath}
$$

where

$$
\bar{X}_{1}, \cdots, \bar{X}_{n},
$$

$$
\bar{X}_{\imath}=\frac{1}{N} \sum_{k=1}^{N} X_{i k} \quad(\imath=1,2, \cdots, n) .
$$

2. The unknown covariance matrix of $\left(X_{1}, \cdots, X_{n}\right)$

$$
\left[\operatorname{Cov}\left(X_{\imath}, X_{j}\right)\right] ; \quad \operatorname{Cov}\left(X_{\imath}, X_{j}\right)=\sum_{i=\imath} \lambda_{i=1}
$$

will be estimated by the sample covariance matrix $\boldsymbol{S}$ or $\boldsymbol{S}^{+}$.

3. And a more refined covariance matrix will be given by $\tilde{\boldsymbol{S}}$.

\section{REFERENCES}

[1] Feller, W., An introduction to probability theory and its applications, Vol. 1, sec. ed. 6-th print (1961).

[2] Kawamura, K., The structure of trivariate Poisson distribution, Vol. 28, No. 1, 1-8 (1976). Kōdai Math. Sem. Rep.

[3] Kendall, M.G. and A. Stuart, The advanced theory of statistics, Vol. 1. Distribution theory, second ed. Criffin (1963).

Department of Mathematics

TOKyo Institute of TECHNOLOgy 